\title{
Gestantes de alto risco: uma análise da autoestima e fatores associados em uma maternidade de referência na cidade do
} Recife, PE, Brasil

High-risk pregnant women: an analysis of self-esteem and associated factors in a reference maternity in the city of Recife, PE, Brasil

Mujeres embarazadas de alto riesgo: un análisis de autoestima y factores asociados en una maternidad de referencia en la ciudad de Recife, PE, Brasil

\section{RESUMO}

A pesquisa tem como objetivo correlacionar com os padrões de autoestima e fatores associados em gestantes de alto risco em uma maternidade de referência na cidade do Recife, Pernambuco, Brasil. Trata-se de um estudo descritivo, transversal, com abordagem quantitativa. A população amostral incluiu 112 mulheres, gestantes, adultas. Na coleta de dados foi utilizado um formulário adaptado da versão no Brasil da Escala de Autoestima de Rosenberg (EAR), acrescido de questões socioeconômicas, obstétricas e reprodutivas. Observou-se que escolaridade foi à única variável com associação significativa com autoestima $(p<0,05)$ e para a referida variável se destaca que o percentual com autoestima insatisfatória reduziu com o grau de escolaridade, sendo $96,0 \%$ entre as que tinham até fundamental incompleto, 75,0\% entre as que ensino fundamental e $61,0 \%$ entre as que tinham ensino médio/superior. Reconhece-se que as maternidades funcionam muitas vezes como porta de acesso para identificação e acolhimento dessas gestantes sendo necessária a atualização/capacitação dos profissionais de saúde para o atendimento adequado e direcionado, uma vez que poucas mulheres têm acesso à avaliação de seu estado emocional durante a gestação.

DESCRITORES: Gestantes; Autoestima; Saúde da Mulher.

\section{ABSTRACT}

The research aims to correlate with self-esteem patterns and associated factors in high-risk pregnant women at a reference maternity hospital in the city of Recife, Pernambuco, Brazil. This is a descriptive, cross-sectional study with a quantitative approach. The sample population included 112 women, pregnant women, adults. For data collection, a form adapted from the Brazilian version of the Rosenberg Self-Esteem Scale (EAR) was used, plus socioeconomic, obstetric, and reproductive issues. It was observed that education was the only variable with a significant association with self-esteem $(p<0.05)$ and for that variable it is highlighted that the percentage with unsatisfactory self-esteem decreased with the level of education, being $96.0 \%$ among those who had even incomplete elementary school, $75.0 \%$ among those with elementary education and $61.0 \%$ among those with secondary / higher education. It is recognized that maternity wards often function as a gateway for the identification and reception of these pregnant women, with the need to update / train health professionals to provide adequate and targeted care, since few women have access to assess their emotional state during pregnancy.

DESCRIPTORS: Pregnant; Self Esteem; Women's Health.

\section{RESUMEN}

La investigación tiene como objetivo correlacionar con patrones de autoestima y factores asociados en mujeres embarazadas de alto riesgo en un hospital de maternidad de referencia en la ciudad de Recife, Pernambuco, Brasil. Se trata de un estudio descriptivo, transversal con enfoque cuantitativo. La población de la muestra incluyó 112 mujeres, mujeres embarazadas, adultos. Para la recolección de datos se utilizó un formulario adaptado de la versión brasileña de la Escala de Autoestima de Rosenberg (EAR), además de aspectos socioeconómicos, obstétricos y reproductivos. Se observó que la educación fue la única variable con asociación significativa con la autoestima $(p<0.05)$ y para esa variable se destaca que el porcentaje con autoestima insatisfac- 


\section{artigo}

Rodrigues da Silva, L.S.; Cordeiro, E.L.; Vasconcelos Neto, A.J.; Alves da Silva, L.C.; Maurício da Silva, A.P.; Gomes, R.R.T.; Vasconcelos Silva, F.M.

Gestantes de alto risco: uma análise da autoestima e fatores associados em uma maternidade de referência na cidade do Recife, PE, Brasil

toria disminuyó con el nivel de educación, siendo 96.0\% entre los que tenían incluso primaria incompleta, 75.0\% entre aquellos con educación primaria y $61.0 \%$ entre aquellos con educación secundaria / superior. Se reconoce que las maternidades suelen funcionar como puerta de entrada para la identificación y recepción de estas gestantes, con la necesidad de actualizar / capacitar a los profesionales de la salud para brindar una atención adecuada y focalizada, ya que pocas mujeres tienen acceso para evaluar su estado emocional durante el embarazo.

DESCRIPTORES: Mujeres Embarazadas; Autoestima; Salud de la Mujer.

RECEBIDO EM: 21/05/2020 APROVADO EM: 22/05/2020

\section{Liniker Scolfild Rodrigues da Silva}

Sanitarista na modalidade residência pela Faculdade de Ciências Médicas (FCM)/Universidade de Pernambuco (UPE). Especialista em Enfermagem Obstetra na modalidade residência pela Faculdade de Enfermagem Nossa Senhora das Graças (FENSG)/ UPE. Recife, Pernambuco (PE), Brasil.

ORCID: 0000-0003-3710-851X

\section{Eliana Lessa Cordeiro}

Mestre em Neuropsiquiatria e Ciências do Comportamento pela Universidade Federal de Pernambuco (UFPE). Recife, Pernambuco (PE), Brasil.

ORCID: 0000-0001-7305-9431

\section{Antonio José de Vasconcelos Neto}

Especialista em Oncologia na modalidade Residência pelo Hospital de Câncer de Pernambuco (HCP)/Instituto de Medicina Integral Prof. ${ }^{\circ}$ Fernando Figueira (IMIP). Recife, Pernambuco (PE), Brasil.

ORCID: 0000-0002-9504-6778

\section{Luana Camila Alves da Silva}

Especialista em Saúde Pública pela Faculdade Alpha. Recife, Pernambuco (PE), Brasil.

ORCID: 0000-0001-5551-7179

\section{Ana Paula Maurício da Silva}

Bacharel em Enfermagem pelo Centro Universitário Maurício de Nassau (UNINASSAU). Recife, Pernambuco (PE), Brasil.

ORCID: 0000-0002-1827-3678

\section{Roberta Rosas Trigueiro Gomes}

Enfermeira, Especialista em Auditoria de Sistemas de Saúde pela Universidade Estácio de Sá.

ORCID: 0000-0002-4341-3441

\section{Fernanda da Mata Vasconcelos Silva}

Doutoranda em Enfermagem pela Universidade de Pernambuco (UPE). Recife, Pernambuco (PE), Brasil.

ORCID: 0000-0001-5465-9714

\section{INTRODUÇÃO}

A gestação é um período peculiar, de transição para as mulheres, devido às adaptações hormonais, psicológicas e sociais, tornando-as mais suscetíveis a eventos estressantes física e mentalmente. Essas situações de desgaste podem resultar em consequências fisiológicas tanto para a mãe quanto para o bebê $\hat{e}^{(1,2)}$. Durante a formação do novo ser no ventre materno, vão sendo recriados sentimentos e percepções acerca de seu próprio corpo. A mulher vai integrando-se em um novo contexto social, onde lhe são imputadas obrigações de acordo com seu novo papel ${ }^{(3)}$.

A mãe é vista como fornecedora dos melhores sentimentos. Porém, em uma grande maioria das vezes é negligenciado que esta nova mulher/mãe, tem necessidades de amor, afeto, cuidado e atenção. $\mathrm{O}$ fato de estar grávida, para muitas, é visto como motivo de contentamento, orgulho e realização, contudo, devido às modificações de percepção corporal, alterações de humor, mudança no padrão do sono, necessidade de adequação aos novos papéis sociais, podem acontecer prejuízos em sua desenvoltura para conduzir sua rotina e papéis habituais, trazendo um decaimento de sua autoapreciação, podendo surgir ou evidenciar transtornos psiquiátricos ${ }^{(4)}$.

$\mathrm{O}$ organismo feminino, durante a gra- 
videz, apesar das alterações anatômicas, endócrinas, hemodinâmicas e imunológicas importantes, consegue manter o equilíbrio através de mecanismos compensatórios e, desta forma, sua evolução, na maior parte dos casos, se dá sem maiores transtornos, sendo classificada como uma gestação de risco habitual. Porém, pode acontecer de em alguns casos, por características específicas hereditárias, ou por algum evento complicador, a evolução acontecer de forma desfavorável, enquadrando-se como gestação de alto risco ${ }^{(5)}$.

A gestação é denominada como de alto risco quando existe qualquer doença materna que afete a condição sociobiológica e possa prejudicar o desenvolvimento da mesma. A necessidade de diversos acompanhamentos médicos, podendo passar por hospitalizações frequentes e procedimentos invasivos geram fatores desencadeantes de sintomas depressivos, como: ansiedade, baixa concentração, medo, irritabilidade, fadiga, inapetência, insônia, dentre outros. Tornando a gestante de alto risco mais suscetível ao desenvolvimento de quadro depressivo( ${ }^{(6)}$.

A autoestima é o juízo de valor que um indivíduo tem de si mesmo, correspondendo ao conjunto de princípios que traz consigo, refletindo no que sente e pensa. É evidenciada através das reações que cada pessoa apresenta frente às diferentes situações e eventos da vida. Quando sua manifestação é positiva, geralmente o indivíduo se sente confiante, competente e possuidor de valor pessoal. A autoestima também é considerada um importante indicador da saúde mental por interferir nas condições afetivas, sociais e psicológicas dos indivíduos, interferindo, assim, na saúde, no bem-estar e na qualidade de vida da população em $\operatorname{geral}^{(7)}$.

Por meio do conceito de autoestima é possível estabelecer distinções nos processos de avaliação da saúde mental da gestante, ajudando a perceber uma autoestima insatisfatória. Com este objetivo, de avaliar a autoestima em um sentindo global, Rosenberg criou sua escala, que ficou conhecida por Escala de Autoestima de Rosenberg (EAR). A escala avalia a atitude e o sentimento positivo ou negativo por si mesmo, onde níveis baixos de autoestima estão relacionados ao aparecimento de transtornos mentais, como depressão, ansiedade e queixas somáticas, o que pode trazer consequências negativas na interação do binômio mãe-bebê, bem como no desenvolvimento individual do ser humano ${ }^{(8)}$.

Desta forma, partindo da observação realística cotidiana da mulher/mãe, surgiu a empatia pela presente temática, tendo em vista que as orientações e cuidados recebidos, na maioria das vezes, têm ação limitada, enfatizando somente uma nova vida em detrimento das necessidades da gestante como ser individual. Esta realidade somada ao fato que no Brasil ainda existem poucos estudos sobre autoestima e qualidade de vida durante a gestação, justifica a importância do presente artigo. Portanto, este estudo tem como objetivo correlacionar com os padrões de autoestima e fatores associados em gestantes de alto risco em uma maternidade de referência na cidade do Recife, Pernambuco, Brasil.

\section{METODOLOGIA}

Trata-se de estudo descritivo, transversal, com abordagem quantitativa realizado na Enfermaria de Alto Risco da Maternidade do Hospital Agamenon Magalhães (HAM), do município de Recife- PE.

A população estudada incluiu mulheres gestantes usuárias da unidade citada. Seguiu-se amostragem do tipo não probabilística, por conveniência, com adoção dos critérios de inclusão: mulheres gestantes que utilizaram os serviços de internação da enfermaria de Alto Risco da Maternidade do HAM durante o período de coleta dos dados, com idade maior de 18 anos. Foram excluídas mulheres gestantes que não apresentaram capacidade de compreensão e/ou de verbalização para responder ao formulário.

Os dados foram coletados durante o período de abril a junho de 2016. Para tanto, utilizou-se um formulário adaptado da versão no Brasil no ano de 2011 da EAR por Hutz ${ }^{(9)}$. Esta é uma medida unidimensional constituída por dez afirmações relacionadas a um conjunto de sentimentos de autoestima e autoaceitação que avalia a autoestima global. Os itens são respondidos em uma escala tipo Likert de quatro pontos variando entre: concordo totalmente $=4$, concordo $=3$, discordo $=2$ e discordo totalmente $=1$. No entanto, metade dos itens é enunciada positivamente, sendo que as opções de resposta representam os números descritos, e a outra metade negativamente, cujos números das respostas devem ser: concordo totalmente $=1$; concordo $=2$; discordo $=3$; discordo totalmente $=4$. Assim, cada item pode receber uma pontuação de no mínimo 1 e no máximo 4. A soma das respostas dos 10 itens fornece o escore da escala, cuja pontuação total oscila de 10 a 40, sendo que a obtenção de uma pontuação alta reflete autoestima elevada. Sendo a autoestima satisfatória definida como escore maior ou igual a 30 na referida Escala. Acrescentada também, questionários sobre as condições socioeconômicas, obstétricas e reprodutivas.

Construiu-se banco de dados no programa Statistics is a Powerful Statistical Software (SPSS) versão 23.0. A análise dos dados foi realizada por meio de estatística descritiva. Para tanto, calculou-se as frequências percentuais e as distribuições de frequência das variáveis analisadas. A análise inferencial foi através do teste Qui-quadrado de Pearson ou Teste Exato de Fisher quando a utilização para o teste de Qui-quadrado não foi verificada. A margem de erro utilizada na decisão dos testes estatísticos foi de 5,0\%. O programa utilizado para digitação dos dados e a elaboração dos cálculos estatísticos foi o SPSS versão 23. A discussão dos resultados fundamentou-se nos pressupostos da autoestima em gestantes de Alto Risco.

$\mathrm{O}$ estudo seguiu as diretrizes regulamentadoras da pesquisa envolvendo seres humanos conforme o preconizado pela Resolução n. ${ }^{\circ}$ 466, de 12 de dezembro de 2012, do Conselho Nacional de Saúde - CNS ${ }^{(10)}$.

As participantes assinaram o Termo de Consentimento Livre Esclarecido 


\section{artigo}

Rodrigues da Silva, L.S.; Cordeiro, E.L.; Vasconcelos Neto, A.J.; Alves da Silva, L.C.; Maurício da Silva, A.P.; Gomes, R.R.T.; Vasconcelos Silva, F.M Gestantes de alto risco: uma análise da autoestima e fatores associados em uma maternidade de referência na cidade do Recife, PE, Brasil

Tabela 1. Caracterização dos antecedentes obstétricos e reprodutivos na vida das gestantes participantes da pesquisa. Recife, PE, Brasil, 2018

\section{Variável}

TOTAL

n

112

$\%$

Idade gestacional

Pré-termo precoce (< 34 semanas)

Pré-termo tardio (34 a 36 semanas e 6 dias)

Termo precoce (37 a 38 semanas e 6 dias)

Termo (39 a 40 semanas e 6 dias)

Número de gestações

Primigesta

Secundigesta

Tercigesta

Multigesta

Número de partos

0

1

2

3 a 8

Cirurgia cesariana em gestações anteriores

Sim

Não

Número de abortos

0

1

2

$>3$

Planejamento da gravidez atual

Planejada

Não planejada

Tabela 2. Distribuição das pesquisadas conforme ocupação e religião. Recife, $\mathrm{PE}$, Brasil, 2018

\begin{tabular}{|c|c|c|c|}
\hline & Variável & $\mathrm{n}$ & $\%$ \\
\hline TOTAL & & 112 & 100,0 \\
\hline \multicolumn{4}{|c|}{ Tem ocupação } \\
\hline Sim & & 76 & 67,9 \\
\hline Não & & 36 & 32,1 \\
\hline \multicolumn{4}{|l|}{ Ocupação } \\
\hline Vendedor & & 2 & 1,8 \\
\hline Autônomo & & 2 & 1,8 \\
\hline Caixa de loja & & 2 & 1,8 \\
\hline
\end{tabular}

(TCLE), assegurando-se o anonimato e o respeito à decisão de não participar ou desistir a qualquer momento do estudo. Obteve aprovação pelo Comitê de Ética e Pesquisa (CEP) do HAM, segundo o Número do Parecer: 2.299.753, e sob CAAE: 5379916.2.000.5197.

É relevante informar que, em virtude deste artigo ser parte do Trabalho de Conclusão de Residência (TCR) do autor Liniker Scolfild Rodrigues da Silva (2017), toda casuística apresentada no presente estudo manteve-se inalterada, sendo utilizada na íntegra, assim como no trabalho original.

\section{RESULTADOS}

A amostra estudada compreendeu 112 mulheres gestantes adultas. Com relação aos indicadores socioeconômicos das participantes, percebe-se predomínio da faixa etária entre 21 a 25 anos (38,4\%), a maioria possui um relacionamento estável com seu parceiro, sendo: do tipo casamento $(36,7 \%)$ e união consensual $(33,95 \%)$. Com relação à prática religiosa, foi frequente a evangélica (50,9\%).

Quanto ao trabalho e rendimento, 40,2\% eram domésticas; $47,3 \%$ tinham rendimento mensal familiar em torno de 1 salário mínimo. Sobre condição de habitação, $82,1 \%$ residiam em zona urbana, $91,1 \%$ moravam em casa. Quanto aos anos de estudo, 42,0\% possuíam nível médio completo.

Com relação aos antecedentes obstétricos e reprodutivos, observa-se que mais da metade já haviam estado grávidas anteriormente (75,0\%). A Tabela 1 apresenta a caracterização dos antecedentes obstétricos e reprodutivos na vida das gestantes participantes da pesquisa. Observa-se que 53,6\% estavam em curso de uma gestação pré-termo precoce. Referente ao quantitativo de gestações prévias, $29,5 \%$ eram tercigestas, $38,4 \%$ já haviam passado por um parto anterior, 59,8\% nunca foram submetidas em algum momento ao parto do tipo cesárea. Dentre a totalidade, a grande maioria $(71,4 \%)$ não tinha aborto anterior. E, por fim, $65,2 \%$ não planejaram 


$\begin{array}{lcc}\text { Costureira } & 4 & 3,6 \\ \text { Doméstica } & 1 & 0,9 \\ \text { Do lar } & 45 & 40,2 \\ \text { Lanchonete } & 1 & 0,9 \\ \text { Professora } & 1 & 0,9 \\ \text { Auxiliar de serviços gerais } & 1 & 0,9 \\ \text { Atendente } & 1 & 0,9 \\ \text { Cozinheira } & 2 & 1,8 \\ \text { Agricultora } & 3 & 2,7 \\ \text { Vigilante } & 1 & 0,9 \\ \text { Agente Comunitária de Saúde (ACS) } & 1 & 0,9 \\ \text { Cabeleireira } & 1 & 0,9 \\ \text { Auxiliar de contábeis } & 1 & 0,9 \\ \text { Call Center } & 1 & 0,9 \\ \text { Educadora de hotelzinho } & 1 & 0,9 \\ \text { Pedagoga } & 1 & 0,9 \\ \text { Cobradora de ônibus } & 1 & 0,9 \\ \text { Técnica de Enfermagem } & 1 & 0,9 \\ \text { Cambista } & 2 & 1,8 \\ \text { Não tem ocupação } & 36 & 32,1 \\ \text { Religião } & & \\ \text { Católica } & 39 & 34,8 \\ \text { Evangélicas } & 57 & 50,9 \\ \text { Sem religião } & 16 & 14,3\end{array}$

Tabela 3. Avaliação das Escalas de Autoestima de Rosenberg. Recife, PE, Brasil, 2018

Variável

TOTAL

n $\%$

$112 \quad 100,0$

Autoestima

Satisfatória

$31 \quad 27,7$

Insatisfatória a gestação atual.

Da Tabela 2 se evidencia que: a maioria $(67,9 \%)$ tinha ocupação e deste percentual a mais frequente $(40,2 \%)$ era do lar e as demais ocupações tiveram percentuais que variaram de $0,9 \%$ correspondendo a uma pesquisada a 3,6\%; aproximadamente a metade $(50,9 \%)$ era evangélicas, seguido de 34,8\% católicas e $14,3 \%$ restante não tinham religião.

Quando questionadas a respeito da autoestima e autoaceitação, conforme a escala de Rosenberg (EAR), que é constituída por dez afirmações ( 5 positivas e 5 negativas), relacionadas a um conjunto de sentimentos, sendo as respostas pontuadas conforme as variantes entre, concordo totalmente, concordo, discordo e discordo totalmente, obteve-se através do cálculo final predomínio de autoestima insatisfatória $(72,3 \%)$, como demonstrado na Tabela 3 que expõe a avaliação das escalas de autoestima de Rosenberg.

Tabela 4 analisa a associação entre a categorização da escala de autoestima com as variáveis sociodemográficas. Nesta tabela se verifica que escolaridade foi à única variável com associação significativa com autoestima $(p<0,05)$ e para a referida variável se destaca que o percentual com autoestima insatisfatória reduziu com o grau de escolaridade, sendo $96,0 \%$ entre as que tinham até fundamental incompleto, $75,0 \%$ entre as que ensino fundamental e $61,0 \%$ entre as que tinham ensino médio/superior.

Não foram registradas associações significativas $(p<0,05)$ entre os resultados da autoestima e os dados obstétricos contidos na Tabela 5 .

Tabela 4. Avaliação da autoestima segundo os dados sociodemográficos. Recife, PE, Brasil, 2018

\section{Autoestima}

Variável

\begin{tabular}{cccccc}
\multicolumn{2}{c}{ Insatisfatória } & \multicolumn{2}{c}{ Satisfatória } & \multicolumn{2}{c}{ Grupo total } \\
$\mathrm{n}$ & $\%$ & $\mathrm{n}$ & $\%$ & $\mathrm{n}$ & $\%$ \\
81 & 72,3 & 31 & 27,7 & 112 & 100,0
\end{tabular}

Faixa etária

18 a 20

21 a 25

26 a 30
10

31

17

58,8
72,

81,0

8,8
72,1
81,0

7
12

41,2

17

100,0 1,00

$$
p(1)=0,662
$$

$4 \quad 19,0 \quad 21 \quad 100,0$




\section{artigo}

Rodrigues da Silva, L.S.; Cordeiro, E.L.; Vasconcelos Neto, A.J.; Alves da Silva, L.C.; Maurício da Silva, A.P.; Gomes, R.R.T.; Vasconcelos Silva, F.M.

Gestantes de alto risco: uma análise da autoestima e fatores associados em uma maternidade de referência na cidade do Recife, PE, Brasil

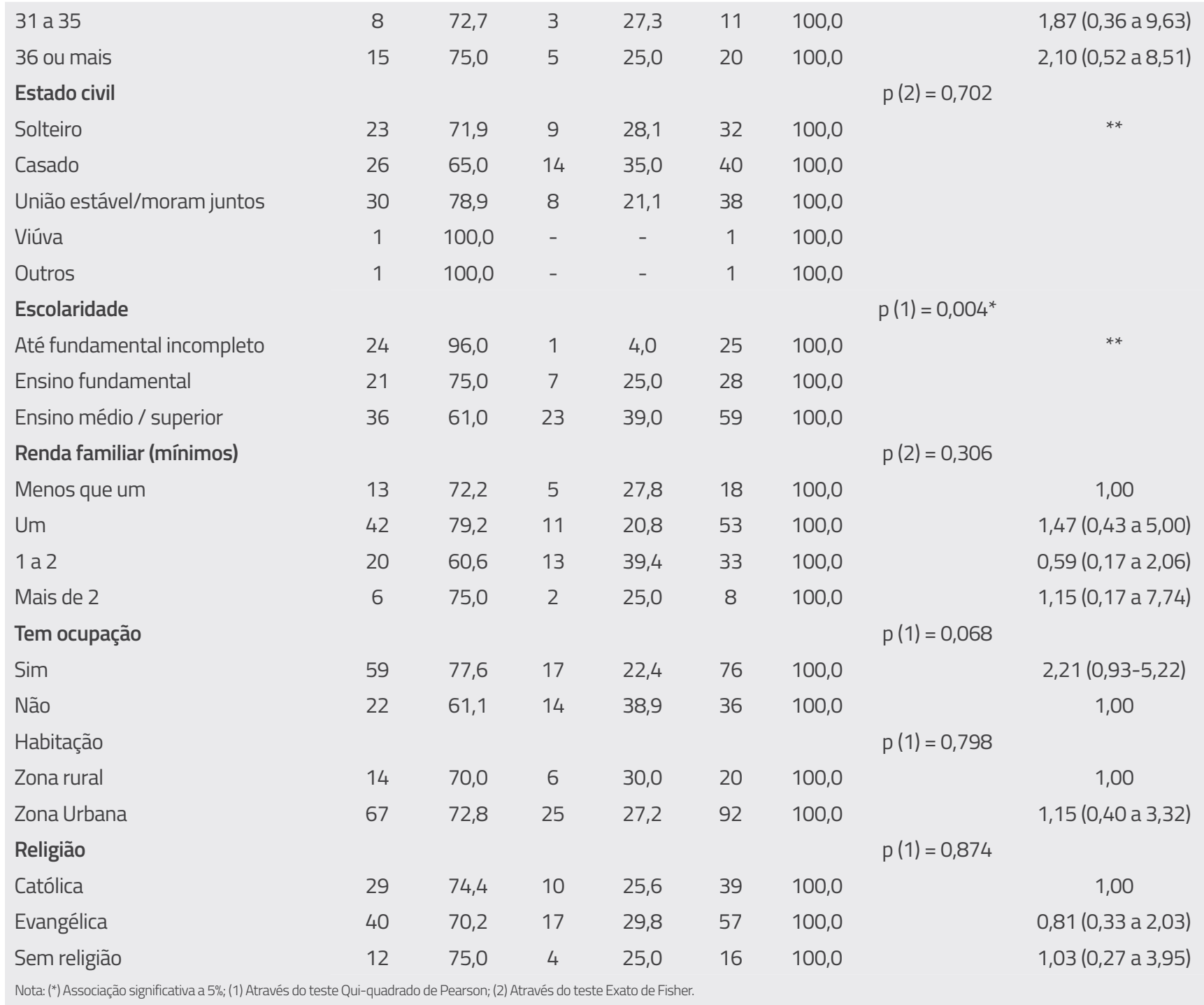

Tabela 5. Avaliação da autoestima segundo os dados obstétricos. Recife, PE, Brasil, 2018

Autoestima

Variável

\begin{tabular}{cccccc}
\multicolumn{2}{c}{ Insatisfatória } & \multicolumn{2}{c}{ Satisfatória } & \multicolumn{2}{c}{ Grupo total } \\
$n$ & $\%$ & $n$ & $\%$ & $n$ & $\%$ \\
81 & 72,3 & 31 & 27,7 & 112 & 100,0
\end{tabular}

TOTAL

Variăvel

Idade gestacional

Pré-termo (Precoce)

Pré-termo (Tardio)

Termo precoce

Termo

Número de gestações

Primigesta

Secundigesta
24

14

20 16
4270,0

2468

485

$-$

$p(2)=0,117$

OR (IC 95\%)

OR (IC $95 \%)$ 


\begin{tabular}{|c|c|c|c|c|c|c|c|c|}
\hline Tercigesta & 25 & 75,8 & 8 & 24,2 & 33 & 100,0 & & $1,25(0,40$ a 3,92$)$ \\
\hline Multigesta & 20 & 87,0 & 3 & 13,0 & 23 & 100,0 & & $2,67(0,62$ a 11,53$)$ \\
\hline Número de partos & & & & & & & $p(2)=0,231$ & \\
\hline 0 & 20 & 71,4 & 8 & 28,6 & 28 & 100,0 & & 1,00 \\
\hline 1 & 27 & 62,8 & 16 & 37,2 & 43 & 100,0 & & $0,68(0,24$ a 1,88$)$ \\
\hline 2 & 21 & 84,0 & 4 & 16,0 & 25 & 100,0 & & $2,10(0,55$ a 8,08$)$ \\
\hline 3 ou mais & 13 & 81,3 & 3 & 18,8 & 16 & 100,0 & & $1,73(0,39$ a 7,76$)$ \\
\hline Número de abortos & & & & & & & $p(2)=0,385$ & \\
\hline Nenhum & 56 & 70,0 & 24 & 30,0 & 80 & 100,0 & & 1,00 \\
\hline Um ou mais & 25 & 78,1 & 7 & 21,9 & 32 & 100,0 & & $1,53(0,58$ a 4,02$)$ \\
\hline $\begin{array}{l}\text { Via de parto das gestações ante- } \\
\text { riores vaginais }\end{array}$ & & & & & & & $p(2)=0,689$ & \\
\hline Sim & 40 & 74,1 & 14 & 25,9 & 54 & 100,0 & & $1,19(0,52$ a 2,72$)$ \\
\hline Não & 41 & 70,7 & 17 & 29,3 & 58 & 100,0 & & 1,00 \\
\hline Número de partos vaginais & & & & & & & $p(2)=0,615$ & \\
\hline 0 & 41 & 70,7 & 17 & 29,3 & 58 & 100,0 & & 1,00 \\
\hline 1 & 23 & 69,7 & 10 & 30,3 & 33 & 100,0 & & $0,95(0,38$ a 2,42) \\
\hline 2 ou mais & 17 & 81,0 & 4 & 19,0 & 21 & 100,0 & & $1,76(0,52$ a 6,01$)$ \\
\hline Cirurgia cesariana & & & & & & & $p(2)=0,844$ & \\
\hline Sim & 33 & 73,3 & 12 & 26,7 & 45 & 100,0 & & $1,09(0,47$ a 2,54$)$ \\
\hline Não & 48 & 71,6 & 19 & 28,4 & 67 & 100,0 & & 1,00 \\
\hline Número de partos cesaria-nos & & & & & & & $p(2)=0,190$ & \\
\hline 0 & 48 & 71,6 & 19 & 28,4 & 67 & 100,0 & & ** \\
\hline 1 & 21 & 65,6 & 11 & 34,4 & 32 & 100,0 & & ** \\
\hline 2 a 3 & 12 & 92,3 & 1 & 7,7 & 13 & 100,0 & & $* *$ \\
\hline Quanto à gravidez & & & & & & & $p(2)=0,725$ & \\
\hline Desejada / planejada & 29 & 74,4 & 10 & 25,6 & 39 & 100,0 & & $1,17(0,49$ a 2,82$)$ \\
\hline Indesejada / não planejada & 52 & 71,2 & 21 & 28,8 & 73 & 100,0 & & 1,00 \\
\hline
\end{tabular}

\section{DISCUSSÃO}

Observa-se que as características das mulheres participantes desse estudo corroboraram com informações de outras pesquisas. Pesquisa publicada em $2015^{(1)}$, investigou a autoestima e qualidade de vida de uma série de gestantes atendidas em unidades do sistema único de saúde do município de Rio Branco - Acre, mostrou que a maioria era maior de 21 anos, possuía o ensino médio completo, rendimento menos que 2 salários mínimos. Semelhantemente, autores ${ }^{(11)}$ descreveram o perfil sociodemográfico da mulher gestante de alto risco, a maioria era casada, ensino médio completo, com faixa etária de 18-40 anos.

Os problemas socioeconômicos presentes na vida da mulher gestante aparecem trazendo consigo inúmeras consequências, como por exemplo: baixa autoestima, depressão, fobia, estresse pós-traumático, entre outros. $\mathrm{E}$ acarretam danos não só na vida da vítima como na vida dos que a cercam e/ou convivem. Neste sentido, os profissionais de saúde, principalmente da atenção primária, devem praticar uma escuta qualificada e o acolhimento para auxiliar sempre que possível a gestante visando minimizar danos ${ }^{(12)}$.

Sobre os antecedentes obstétricos, estu- $\mathrm{do}^{(13)}$ apontou que a maioria das gestantes de alto risco eram multigestas $(65,5 \%)$, não referiam aborto prévio $(73,1 \%)$, já possuíam ao menos um filho $(34,6 \%)$, via de parto cesárea $(59,6 \%)$, e curso de gestação pré-termo precoce (71,2\%). Resultados que corroboram com nosso estudo, divergindo somente em relação ao número de gestações anteriores e a via de parto.

Os resultados revelaram gestante de alto risco com predomínio de autoestima insatisfatória (72,3\%), concordando com os resultados de outros estudos nos quais apontaram um elevado número de mulheres gestantes que compóem o grupo de alto risco com baixos níveis de autoestima, elas 
apresentam sentimento de impotência, desespero, distorção da autoestima. Também se preocupam com a possível condição de vida diferenciada do seu filho prematuro, marcada pelas limitações, cuidados especiais e muitas vezes preconceitos ${ }^{(14)}$.

Estes resultados evidenciam, portanto, que uma gestação traz consigo diversos desafios a serem sentidos e vivenciados, ainda mais tratando-se de um alto risco, envolvendo o contexto mãe-mulher-sociedade como um todo. Não raro, sentem insegurança, timidez, frustração e medo do desconhecido. É perceptível, ainda, distorções entre os papéis de mulher e mãe, pois corriqueiramente esta relação ocorre com a desvalorização do ser mulher, levando à diminuição da vontade de cuidar da aparência, de arrumar-se, ou, até mesmo, do desconhecimento das alteraçóes de seu corpo no processo gestacional ${ }^{(15)}$.

Semelhantemente com os resultados encontrados, temos um estudo onde mais da metade de suas gestantes entrevistadas $(60,6 \%)$ apresentou autoestima insatisfatória ${ }^{(16)}$. Outro estudo comparativo entre gravidez habitual e de alto risco expressa índices mais elevados para gestantes de alto risco, pelo menos $56,5 \%$ das gestantes de alto risco demonstraram ao menos índices mínimos de depressão. Essa dinâmica comportamental é promovida principalmente por medo de má formação do feto, risco de morte e sentimento de incompetência em seu papel de procriadora ${ }^{(6)}$.

É válido observar que a diminuição da autoestima obedece a uma escala progressiva, iniciando com agressões depreciação de si mesmo que passa para sentimento de inutilidade, podendo chegar à depressão. E é por isso que é essencial o acompanhamento, sempre que possível, da psicologia. Neste sentido, o auxilio deste profissional visa reduzir riscos psicossociais presentes no processo gravídico ${ }^{(17)}$.

Quando cruzados os dados socioeconômicos com os níveis de autoestima, temos que análogo ao nosso estudo, estudo ${ }^{(4)}$ caracterizou a mulher gestante portadora de depressão como jovem (20 a 25 anos), ensino médio completo $(37,3 \%)$, tinha alguma atividade remunerada (49,3\%), prática religiosa

católica (56,9\%), discordando neste último tópico dos resultados encontrados no presente estudo, que apresenta predomínio de religião evangélica. Um estudo constatou que a maior parte das mulheres com baixos níveis de autoestima era casada ou vivia em união estável com o companheiro ${ }^{(16)}$.

Em referência à prática religiosa, é válido mencionar que, durante o processo de gestação, a mulher passa por situações conflituosas, dessa forma, é notável a busca de meios de enfrentamento dessas situações. Neste contexto, integra-se a prática religiosa, atuando como forma de escape, independentemente de sua denominação ${ }^{(4)}$.

Constatou-se que

a maior parte das

gestantes de alto

risco apresentou

autoestima

insatisfatória...

Observou-se que o nível de escolaridade apresentou um valor significativo na relação com uma autoestima insatisfatória, igualmente, estudo realizado para avaliar a autoestima das gestantes com uso da escala de autoestima de Rosenberg encontrou relação significativa entre baixa escolaridade e autoestima insatisfatória, $\mathrm{p}=0,04^{(16)}$. Autores ${ }^{(11)}$ também encontraram os mesmos resultados com prejuízo da autoestima em gestante com escolaridade até o ensino médio.

Quando realizada associação dos dados obstétricos com os níveis de autoestima, não houve associações significativas, contudo, foram verificados em outros estudos idênticos ao nosso. Estudos estes que caracterizaram em sua pesquisa que a autoestima insatisfatória foi significativamente mais comuns entre as mães de partos prematuros em comparação com as mães de recém-nascidos a termo e que o risco de depressão em mães de bebês prematuros foi duas vezes maior que o risco em mães de bebês a termo ${ }^{(18,19)}$. Estudo ${ }^{(20)}$ apresentou um perfil obstétrico onde, $48 \%$ das mulheres estavam na segunda ou terceira gestação, 38,8\% afirmaram histórico de aborto, e 51,4\% não desejaram ou planejaram a gestação, dados estes que corroboram com nosso estudo, divergindo apenas no tocantes a gravidez desejada.

\section{CONCLUSÃO}

Constatou-se que a maior parte das gestantes de alto risco apresentou autoestima insatisfatória, manifestando sintomas autodepreciativos a acerca de seu próprio corpo e sentimentos negativos em relação ao desenvolvimento da gestação, como: medo, insegurança, tornando-as mais suscetíveis ao desenvolvimento de transtornos psiquiátricos.

Os eventos depreciativos da autoestima nas gestantes não é um fenômeno novo, no entanto, observa-se que a mesma alcança números preocupantes e apresenta raízes no sistema patriarcal, acarretando danos à qualidade de vida e saúde das mulheres. É preciso interromper esta dinâmica cultural na qual a mulher é cobrada como responsável total pelo equilíbrio familiar.

Faz-se necessário, portanto, repensar a divisão sexual e social das responsabilidades familiares e desnaturalizar a dominação sobre mulher, principalmente a gestante permitindo-a ser contemplada como ser em processo de transformação e adaptação, fornecendo-a as bases para o seu desenvolvimento emocional e físico.

Em suma, reconhece-se que a atenção em saúde, dispensada nas maternidades, é em algumas vezes a porta de acesso para identificação e acolhimento de mulheres em situação de transtornos emocionais e/ ou psiquiátricos. Neste sentido, recomen- 
da-se promover a atualização/capacitação dos profissionais de saúde das maternidades (sobretudo as de alto risco), aqui em espe- cial do enfermeiro, para que possam reconhecer as vulnerabilidades das gestantes de alto risco e intervir com qualidade por meio da abordagem psicossocial, educativa e clínico-preventiva no atendimento às mulheres em situação de vulnerabilidade.

\section{REFERÊNCIAS}

1. Santos $A B$, et al. Autoestima e qualidade de vida de uma série de gestantes atendidas em rede pública de saúde. Revista Cogitare Enfermagem [Internet]. 2015 [acesso em 13 out 2017];20(2):392400. Disponivel em: http://revistas.ufpr.br/cogitare/article/ view/38166

2. Zanatta E, Pereira Caroline RR, Alves AP. A experiência da maternidade pela primeira vez: as mudanças vivenciadas no tornar-se mãe. Pesqui. prát. Psicossociais [Internet]. 2018 [acesso em 20 mai 2020];13(1):1-16. Disponível em http://pepsic.bvsalud.org/scielo.php?script=sci_arttext\&pi$d=S 1809-89082018000100005 \& \operatorname{lng}=p t \& n r m=i s o$.

3. Tomaschoewski-Barlem JG, et al. Promovendo A Autoestima Na Gestação: foco no acolhimento. Enfermagem em Foco. 2016;7(2):83-86. http://dx.doi.org/10.21675/2357-707x.2016 v7.n2.801.

4. Silva MMJ, et al. Ansiedade e depressão na gravidez: caracterização de gestantes que realizaram pré-natal em unidades públicas de saúde. Revista de Enfermagem UFPE on line. 2015;9(7):9027-37. Doi: 10.5205/reuol.8074-70954-1-SM0907supl201512.

5. Tostes NA, Seidl EMF. Expectativas de Gestantes sobre o Parto e suas Percepções acerca da Preparação para o Parto. Trends in Psychology [Internet]. 2016 [acesso em 20 dez 2017];24(2):681-693. Disponível em: http://pepsic.bvsalud.org/pdf/tp/v24n2/v24n2a15. pdf.

6. Zeoti FS, Petean EBL. Apego materno-fetal, ansiedade e depressão em gestantes com gravidez normal e de risco: estudo comparativo. Estudos de Psicologia, Campinas [Internet]. 2015 out. [acesso em 20 dez 2017];32(4):675-683. Disponivel em: http://www.scielo. br/scielo.php?pid=S0103-166X2015000400675\&script=sci_abstract\&tlng=pt.

7. Cabral AMF. Avaliação da autoestima e qualidade de vida dos fiIhos separados pela hanseníase no Estado do Rio Grande do Norte. 2016. 86 f. Tese (Doutorado) - Curso de Enfermagem na Atenção à Saúde, Centro de Ciências da Saúde, Universidade Federal do Rio Grande do Norte, Natal, 2016.

8. Sena R, et al. Correlação entre imagem corporal e autoestima em pessoas com estomias intestinais. Psic., Saúde \& Doenças [Internet]. 2018 dez. [acesso em 20 mai 2020];19(3):578-590. Disponível em: http://www.scielo.mec.pt/scielo.php?script=sci_arttext\&pi$\mathrm{d}=\mathrm{S} 1645-00862018000300009 \&$ Ing=pt\&nrm=iso.

9. Hutz CS, Zanon C. Revisão da adaptação, validação e normatização da escala de autoestima de Rosenberg. Revista Avaliação psicológica [Internet]. 2011 [acesso em 15 jan 2017];10(1):41-49. Disponível em: http://pepsic.bvsalud.org/scielo.php?script=sci_arttext\&pid=S1677-04712011000100005.

10. Conselho Nacional de Saúde (BR). Resolução n. ${ }^{\circ}$ 466, 12 de dezembro de 2012 [Internet]. Diário Oficial da União, Poder Executivo, Brasilia, DF, 13 jun. 2013 [acesso em 15 dez 2016]. Seção 1. p.59-62. Disponivel em: http://sintse.tse.jus.br/documentos/2013/ Jun/13/cns-resolucao-no-466-de-12-de-dezembro-de-2012.

11. Guerra JVV, Valete COS, Alves VH. Socio-demographic and health profile of pregnant women in a high-risk prenatal care. Braz. J. Hea. Rev [Internet]. 2019 fev. [acesso em 25 mai 2020];2(1):249261. Disponivel em: https://www.brazilianjournals.com/index.php/ BJHR/article/view/911.

12. Oliveira EC, Barbosa SM, Melo SEP. The Importance Of Monitoring Prenatal Made By Nurses. Revista Científica Facmais [Internet]. 2016 jul. [acesso em 25 ago 2020];7(3):24-38. Disponivel em: https://docplayer.com.br/53056080-A-importancia-do-acompanhamento-pre-natal-realizado-por-enfermeiros-the-importance-of-monitoring-prenatal-made-by-nurses.html.

13. Luz BG, et al. O perfil das gestantes de alto risco acompanhadas no pré-natal da policlínica de Divinópolis-MG, no biênio 2013/14 Journal Of Health \& Biological Sciences. 2015;3(3):137-128. http:// dx.doi.org/10.12662/2317-3076jhbs.v3i3.177.p137-143.2015.

14. Ulloque-Caamano L, Monterrosa-Castro A, Arteta-Acosta C. Prevalencia de baja autoestima y nivel de resiliencia bajo, en gestantes adolescentes de poblaciones del caribe colombiano. Rev. chil. obstet. Ginecol. 2015 dez.;80(6):462-474. http://dx.doi. org/10.4067/S0717-75262015000600006.

15. Silva MLFS. Gravidez de alto risco: adaptação psicológica de gestantes. Revista Saúde [Internet]. 2016 [acesso em 15 out 2017];10(1). Disponível em: http://revistas.ung.br/index.php/saude/article/view/2593.

16. Carvalho IG, et al. Ansiedade, depressão, resiliência e autoestima em indivíduos com doenças cardiovasculares. Rev. Latino-Am. Enfermagem [Internet]. 2016 [acesso em 20 mai 2020];24:e2836. Disponivel em: http://www.scielo.br/scielo.php?script=sci_arttext\&pid=S0104-11692016000100432\&Ing=en\&nrm=iso.

17. Paiva TT, Pimentel CE, Moura GB. Violência conjugal e suas relações com autoestima, personalidade e satisfação com a vida. Gerais, Rev. Interinst. Psicol. [Internet]. 2017 dez. [acesso em 20 mai 2020];10(2):215-227. Disponível em http://pepsic.bvsalud.org/scielo.php?script=sci_arttext\&pi$d=S 1983-82202017000200007 \&$ Ing=pt\&nrm=iso.

18. Candido TCR, et al. O uso de bebida alcoólica entre gestantes adolescentes. SMAD, Rev. Eletrônica Saúde Mental Álcool Drog. (Ed. port.). 2019 dez.;15(4):1-8. http://dx.doi.org/10.11606/issn.18066976.smad.2019.151701.

19. Cordeiro EAO, Silva LMS, Silva GL. Self-esteem in pregnancy: prevention and Social Protection. Revista Cientifica do Unisalesiano. 2018 dez:;9(19):427-439.

20. Novaes ES, et al. Perfil obstétrico de usuárias do sistema único de saúde após implantação da rede mãe paranaense. Revista Ciência Cuidado e Saúde. 2015 dez.; 14(4):1436-1442. http://dx.doi. org/10.4025/cienccuidsaude. 